\title{
Aufsatz
}

\section{Oliver Thränert*}

\section{Aktuelle nukleare Gefahren und die Probleme der Rüstungskontrolle}

https://doi.org/10.1515/sirius-2018-4003

Kurzfassung: Atomare Gefahren wachsen als Folge der gegenwärtigen Großmächtekonkurrenz, wobei weder die USA, Russland noch China bereit sind, hinsichtlich der Kontrolle atomarer Rüstung politische Führungsverantwortung zu übernehmen. Auch die übrigen Kernwaffenmächte denken weniger an Rüstungskontrolle, sondern setzen auf die Modernisierung oder gar den Ausbau ihrer nuklearen Arsenale. Diese Bemühungen hängen immer mehr mit neuen Entwicklungen bei der Raketenabwehr oder Cyberfähigkeiten zusammen, was zusätzliche Unsicherheitsfaktoren schafft. Dieser Beitrag sucht zu erklären, warum nukleare Rüstungskontrolle nach Ende des Kalten Krieges nahezu zum Erliegen gekommen ist und argumentiert, dass dieses Konzept angesichts dieser globalen Entwicklungen wieder in den Vordergrund rücken sollte. Europa ist besonders gefordert, bezüglich strategischer Stabilität neue Denkanstöße zu erarbeiten und den Diskurs über die Neubelebung der nuklearen Rüstungskontrolle neu zu initiieren.

Schlüsselbegriffe: Kernwaffen, USA, Russland, China, nukleare Proliferation, Rüstungskontrolle, Nichtverbreitungspolitik

Abstract: Nuclear risks are increasing due to current great power rivalry. However, neither the United States, Russia nor China are willing to take over political responsibility when it comes to the control of nuclear armaments. In addition, other nuclear powers do not focus on nuclear arms control either, but rather focus on the modernization or even expansion of their nuclear arsenal. These efforts are closely related to new developments in missile defense and capabilities in the field of cyber, which create further security concerns. This article aims at explaining why nuclear arms control has been stopped after the end of the Cold War and argues in favor of the revival of nuclear arms control as a strategic concept. Europe is especially called upon in taking the initiative towards a new arms control discourse.

*Kontakt: Dr. Oliver Thränert, Leiter des Think Tanks am Center for Security Studies der ETH Zürich;

E-Mail: oliver.thraenert@sipo.gess.ethz.ch
Keywords: Nuclear weapons, United States, Russia, China, nuclear proliferation, arms control, non-proliferation

\section{Einleitung}

Die Entstehung des nuklearen Zeitalters ging einher mit der Herausbildung einer bipolaren internationalen Ordnung. Die Furcht vor einem nuklearen Armageddon führte nach der mit Glück überstandenen Kuba-Krise 1962 zu kooperativen Anstrengungen der beiden Antipoden USA und Sowjetunion mit dem Ziel, trotz bestehender strategischer Konkurrenz das gemeinsame Überleben zu sichern. Die bipolare Konstellation ist mittlerweile längst einer Welt gewichen, die in mehrere Machtzentren zerfällt. Großmächtekonkurrenz ist das hervorstechende Merkmal der heutigen internationalen Arena. Zugleich fokussiert sich der bisherige Anwalt einer liberalen Weltordnung, die USA, ohne deren Führungsleistung die Kontrolle nuklearer Rüstungen während des Kalten Krieges gar nicht denkbar gewesen wäre, auf sich selbst und auf eng definierte nationale Interessen. Ebenso zeigt Russland keinerlei Interesse an einer regelbasierten nuklearen Ordnung. Mehr noch: Der Kreml verstösst mittlerweile gegen Eckpfeiler der europäischen Sicherheitsordnung, wie dem Verbot landgestützter atomarer Mittelstreckenwaffen (INF-Vertrag). Das an internationalem Gewicht gewinnende China ist unwillig oder unfähig, die vormalige Führungsrolle der USA hinsichtlich der Kontrolle atomarer Rüstungen und der Verantwortung für das gemeinsame Überleben zu übernehmen. Es ist zweifelhaft, ob der chinesischen Führung überhaupt die Logik von Rüstungskontrolle einleuchtend ist, die sich an gemeinsamen Sicherheitszielen orientiert und darauf aufbauend versucht Regeln zu entwickeln, die alle Seiten binden.

Indessen wachsen die atomaren Gefahren. Alle neun Kernwaffenmächte modernisieren ihre nuklearen Rüstungen oder bauen diese aus. Neue technologische Entwicklungen wie eine verbesserte Zielgenauigkeit von Raketen oder moderne Möglichkeiten zum Aufspüren von U-Booten gefährden die Überlebensfähigkeit atomarer Arsenale. Zudem können nukleare strategische Fragen nicht mehr 
isoliert betrachtet werden, hängen sie doch immer mehr mit Entwicklungen bei der Raketenabwehr oder neuen Cyberfähigkeiten zusammen. Das nukleare Gleichgewicht ist dadurch gefährdet. Die im Kalten Krieg geltende Formel „Wer zuerst schießt, stirbt als Zweiter“ könnte bald ausgehebelt werden.

Sowohl die Politik wie auch die Öffentlichkeit in westlichen Ländern reagieren auf diese immer bedrohlichere Entwicklung zumeist mit Ratlosigkeit. Sofern es überhaupt eine Debatte gibt, ist sie zumeist geprägt von hilflos wirkenden Rufen nach einer Rückkehr zu Abrüstung und Rüstungskontrolle. Andere flüchten sich in unrealistische Maximallösungen und unterstützen die seit September 2017 zur Unterzeichnung aufliegende Kernwaffenverbotskonvention. Diese bietet jedoch keine Antworten auf die entscheidende Frage nach einer erforderlichen lückenlosen Überwachung eines solchen Verbots. ${ }^{1}$ Entsprechend wird das Abkommen von allen Atomwaffenstaaten und allen denjenigen Ländern, die unter dem atomaren Schutz der USA stehen, boykottiert.

Die Frage ist, welche Rolle kann Rüstungskontrolle in dieser Lage spielen? Das Konzept der Rüstungskontrolle wurde gegen Ende der 50er Jahre und zu Beginn der 60er Jahre des vergangenen Jahrhunderts entwickelt. Oberstes Ziel war die Verhinderung umfassender Nuklearkriege und die Herstellung strategischer Stabilität. Dieses Konzept ist nach dem Ende des Kalten Krieges weitgehend in den Hintergrund gerückt. Es lohnt, dieses Konzeptes heute wieder aufzugreifen, denn es bleibt weiterhin wichtig, über politische Instrumente zu verfügen, die es erlauben, gefährliche nukleare Dynamiken zu verhindern.

In einem ersten Schritt werden die Grundgedanken der Rüstungskontrolle und ihre Wirkung in der politischen Praxis kurz skizziert. In einem zweiten Schritt werden diejenigen Faktoren herausgearbeitet, die die heutige Situation vom Kalten Krieg unterscheiden. Eine solche Analyse ist die zwingende Voraussetzung, um Rüstungskontrolle auf die heutige Zeit anzupassen. Entsprechend liegt der Schwerpunkt dieses Beitrages auf dem Versuch, besser verständlich $\mathrm{zu}$ machen, wo wir heute stehen, anstatt vorschnell Vorschläge nach dem Wohin zu unterbreiten.

Die Möglichkeiten Deutschlands und Europas auf dem Feld der nuklearen Rüstungskontrolle sind begrenzt. Dennoch muss der alte Kontinent in Ermangelung amerikanischer Führung wenigstens versuchen, Denkanstöße zu geben. Deutschland ist gefordert, mit europäischen und weiteren gleichgesinnten Partnern einen Diskurs über die Neubelebung der nuklearen Rüstungskontrolle zu ini-

1 Vgl. Roberts 2018. tiieren. Auch wenn es einer völligen Überforderung gleichkäme, von Deutschland und seinen Partnern allein eine Kehrtwende der derzeit konzeptionslos dahintaumelnden atomaren Rüstungskontrollpolitik zu erwarten, gilt, dass die Zeit des bloßen Bewahrens und Verwaltens einst geschlossener Abkommen vorbei sein muss. Stattdessen sollten neue nukleare Rüstungskontrollinitiativen wieder Bestandteil des strategischen Denkens und Handelns werden.

\section{Rüstungskontrolle: Konzept und politische Praxis}

Nukleare Rüstungskontrolle ist der Versuch, die Risiken nuklearer Waffen und auch nuklearer Abschreckung einzuhegen. Das Konzept basiert auf Überlegungen, die unmittelbar nach dem Ende des Zweiten Weltkrieges und dem Abwurf von Atomwaffen auf die beiden japanischen Städte Hiroshima und Nagasaki in den USA entwickelt worden waren. Diese gehen davon aus, dass im Zeitalter nuklearer Waffen grundlegende Annahmen über Krieg und Frieden revidiert werden müssen. Auch wenn Bernard Brodie eher Strategieforscher denn Rüstungskontrollexperte war, formulierte er bereits 1946, was auch maßgeblich für die Rüstungskontrolle werden sollte: „Thus far the chief purpose of our military establishment has been to win wars. From now on its chief purpose must be to avert them. It can have almost no other useful purpose. “2

Rüstungskontrolle ist nicht identisch mit nuklearer Abrüstung. Letztere ist zwar wünschenswert, schon um überflüssige Kosten für atomare Rüstungen zu vermeiden, aber absehbar nicht realisierbar. Übergeordnetes Ziel der nuklearen Rüstungskontrolle ist die Herstellung und Erhaltung von Stabilität, besonders in Krisensituationen. Prinzipiell können sowohl Ab-, Um- oder sogar Aufrüstungsschritte zur Stabilisierung der Abschreckungssituation beitragen. Ungeachtet politischer Konfrontationen geht die Rüstungskontrolle von dem gemeinsamen Interesse von Staaten aus, umfassende Nuklearkriege zu vermeiden. Ziele der nuklearen Rüstungskontrolle sind daher die Vermeidung von Anreizen zur nuklearen Präemption, die Herstellung eines Instrumentariums zum Krisenmanagement, technische und sicherheitsbezogene Maßnahmen zur Verhinderung ungewollter Kriege oder von Kriegen durch Unfall sowie die Schadensbegrenzung für den Fall, dass ein Krieg dennoch ausbricht. Die revolu-

2 Zitiert bei Brodie 1978, 65. Zu den Hintergründen vgl. Krause 2011, 414 f. sowie Steiner 1991. 
tionäre Einsicht, wonach die Sicherheit des Gegners für die eigene Sicherheit immer mitzudenken ist, bildet den Kern des Rüstungskontrollkonzepts. Die Basis dafür ist das wohl verstandene Eigeninteresse: Unsicherheit beim Gegner kann in Krisensituationen in ungewollter Eskalation münden. Vertreter der nuklearen Rüstungskontrolle sahen daher die Existenz beiderseitiger überlebensfähiger nuklearer Zweitschlagpotenziale als zentral für die Herstellung und Aufrechterhaltung strategischer Stabilität an.

Rüstungskontrolle schließt eine vollständige nukleare Abrüstung nicht von vornherein aus. Da sich die Abrüstung aber am Kriterium der Stabilität orientieren muss, müsste in einer Welt ohne Kernwaffen sichergestellt werden, dass es auch dabei bleibt und es nicht zu unkontrollierten, schnellen nuklearen Wiederaufrüstungen kommt, die atomare Kriege wahrscheinlicher machen können als in einer Situation der gegenseitigen nuklearen Zweitschlagfähigkeit. Es bedarf also eines umfassenden und extrem effektiven Verifikationssystems, auf das sich alle Staaten verlassen können. Selbst dann bliebe allerdings die Frage, wie entdeckte nukleare Wiederaufrüstung von den anderen, nicht nuklear bewaffneten Staaten wirksam sanktioniert werden könnte. Oder die Staatenwelt würde zugunsten einer Weltregierung aufgelöst. Da beide Wege bis auf weiteres nicht beschritten werden können, muss es aus Sicht der Rüstungskontrolle um Stabilität in einer Welt mit Kernwaffen gehen. ${ }^{3}$

In der politischen Praxis konnten mittels Rüstungskontrolle einige durchaus vorzeigbare Ergebnisse erzielt werden, die zur Stabilisierung der nuklearen Abschreckung beitrugen. In mehreren Verträgen zwischen den USA und der Sowjetunion und später Russland wurden Obergrenzen für die strategischen Kernwaffen beider Seiten vereinbart, die zum Teil mit zahlenmäßigen Reduzierungen einhergingen. Wichtig war zudem, dass besonders schwere Atomwaffen und die Anzahl von Mehrfachsprengköpfen reduziert wurden. Bei den nuklearen Mittelstreckenwaffen gelang 1987 gar ein komplettes Verbot, sodass eine destabilisierende Rüstungsdynamik in diesem Waffenbereich vermieden werden konnte. Weitere nicht-strategische Nuklearwaffen wurden nach Ende des Kalten Krieges in einseitigen, jedoch abgesprochenen Schritten massiv reduziert. Mit dem ABM-Vertrag von 1972 einigten sich beide Seiten auf Beschränkungen der strategischen Raketenabwehr, sodass ein Wettlauf zwischen Offensivund Defensivsystemen ausblieb. Nach dem Zerfall der Sowjetunion bot das START-Abkommen von 1991 über die Begrenzung strategischer Kernwaffen die Möglichkeit,

3 Vgl. zum Konzept der Rüstungskontrolle grundlegend: Forndran 1970, siehe auch Wohlstetter 1959, Schelling 1966 und Kissinger 1974. dass Weißrussland, die Ukraine und Kasachstan sich der auf ihren Territorien befindlichen Atomwaffen entledigten und 1994 Russland übergaben. ${ }^{4}$

Vor allem aber trugen der permanente Dialog und die gemeinsam überprüfte Umsetzung von Vereinbarungen zur Bildung von Transparenz und Vertrauen bei. Das Denken der jeweiligen Gegenseite wurde besser verständlich. Sowjetische Offizielle, darunter auch Militärs, gaben als Konsequenz ihres permanenten Atomdiskurses mit ihren amerikanischen Verhandlungspartnern ihre vom Marxismus-Leninismus geprägte Vorstellung auf, wonach der Sozialismus den Imperialismus in einem großen Krieg gesetzmäßig besiegen würde, und schlussfolgerten, dass es in einem großen Kernwaffenkrieg keine Sieger geben könne. ${ }^{5}$ Auch wenn es auf westlicher Seite Experten gab, die eine Siegmöglichkeit auch in einem Atomkrieg als Element einer wirksamen atomaren Abschreckung ansahen, ${ }^{6}$ überwog doch die Einsicht, dass eine große nukleare Auseinandersetzung unbedingt vermieden werden sollte. Obwohl - wie Kritiker nicht müde wurden $\mathrm{zu}$ bemängeln ${ }^{7}$ - die Weiterentwicklung von Kernwaffen nie gänzlich gestoppt wurde, entwickelte sich eine gemeinsame Verantwortung für die Verhinderung eines allgemeinen atomaren Untergangs.

Wo stehen wir mit der Rüstungskontrolle heute? Das Ende des Kalten Krieges führte zu einem Bedeutungsverlust der nuklearen Rüstungskontrolle. Auf strategischer Ebene begrenzte zwar der 1991 unterschriebene und 1994 in Kraft getretene START-Vertrag die strategischen Kernwaffen Amerikas und Russlands. Diesem folgte 2010 das Neu-START-Abkommen. Die politische Aufmerksamkeit verlagerte sich jedoch auf andere Themen wie nukleare Weiterverbreitung oder auch Nuklearterrorismus. In der Tat wurde Russland nicht mehr als Gegner, sondern als Partner der USA und der NATO angesehen.

Seitdem geriet die nukleare Rüstungskontrolle nicht nur aus dem Fokus. Sie befindet sich bereits seit geraumer Zeit in einer formidablen Krise, die bereits in einer schrittweisen Rückabwicklung des während des Kalten Krieges Erreichten resultierte. So kündigte US-Präsident George W. Bush mit Wirkung vom Juni 2002 den ABMVertrag; seit Jahren werfen sich die USA und Russland gegenseitig die Nichteinhaltung des INF-Vertrages vor. Im Oktober 2018 kündigte US-Präsident Doanld Trump daher an, das Abkommen verlasssen zu wollen. Russland

4 Vgl. Woolf/Kerr/Nikitin 2018.

5 Vgl. Arbatov 2017.

6 Vgl. Gray/Payne 1980.

7 Vgl. zur die klassische Kritik an der Rüstungskontrolle Senghaas 1972. 
bezichtigt die USA der Nichteinhaltung des Neu-START Vertrages, die Zukunft dieses Abkommens ist also ebenfalls nicht gesichert; und derzeit machen Washington und Moskau keinerlei Anstalten, dieses Neu-START, das den strategischen Nuklearwaffen beider Seiten Begrenzungen auferlegt und das 2021 auslaufen wird, durch ein neues Abkommen zu ersetzen. Zugleich verschlechterten sich die bilateralen Beziehungen schrittweise und spätestens seit 2014 und der russischen Annexion der Krim sind Russland und die USA wieder zu Gegnern geworden. Das Bewusstsein der gemeinsamen Verantwortung für das Überleben im Nuklearzeitalter ist hingegen nicht wiederbelebt worden.

\section{Aktuelle nukleare Entwicklungen}

Vielmehr gewinnt man den Eindruck, dass die nukleare Konkurrenz zwischen den USA und der NATO einerseits und Russlands andererseits sich zuspitzt. Beide Seiten führen neue Kernwaffen ein oder planen dies und auf beiden Seiten nehmen die Vorhaben der jeweiligen anderen Seite bei den eigenen Planungen einen breiten Raum ein. Donald Trump führt die Politik Barack Obamas, die Bedeutung der Atomwaffen für die amerikanische Sicherheit möglichst zu minimieren, nicht fort. Russland hat Obamas Anliegen nie geteilt. Präsident Vladimir Putin lässt kaum eine Gelegenheit aus, um auf die Kernwaffen als wichtiges russisches Großmachtattribut hinzuweisen.

Die USA wollen auch unter Präsident Donald Trump ihre nukleare strategische Triade bestehend aus land- und seegestützten ballistischen Raketen sowie Fernbombern und luftgestützten Marschflugkörpern beibehalten. Da die entsprechenden Trägersysteme inzwischen in die Jahre gekommen sind, stehen in allen Bereichen umfassende Neubeschaffungen an. So werden die U-Boote der Ohio-Klasse durch neue der Columbia-Klasse ersetzt. Die landgestützten Minuteman-Raketen werden ebenfalls von einem neuen System abgelöst, wobei jede Rakete nur einen Sprengkopf tragen soll. Ferner sollen die B-52H-Bomber, ausgestattet mit luftgestützten Marschflugkörpern, sowie die B-2A-Bomber zugunsten des neuen B-21-Bombers ausgemustert werden. Die Luftflotte wird zudem einen weit reichenden, neuen Marschflugkörper erhalten. Überdies sollen die in Europa stationierten, konventionell wie nuklear einsetzbaren Kampfflugzeuge der Typen F-15E und F-16 durch F-35A ersetzt werden. Die Gesamtanzahl der amerikanischen Kernwaffen soll sich zwar nicht erhöhen, aber auch bei den nuklearen Sprengköpfen stehen Moder- nisierungen an. Eine kleine Anzahl nuklearer Sprengköpfe für seegestützte Raketen soll mit einer Option für den Einsatz mit geringerer Sprengkraft ausgerüstet werden. Dies soll die Rückversicherung der US-Alliierten in Europa wie auch in Asien im Angesicht der Bedrohungen aus Russland, China und Nordkorea stärken. Kritiker befürchten jedoch, dass durch „kleine“ Atomwaffen die Schwelle zu ihrem Einsatz sinken könnte. ${ }^{8}$

Russland hat die Modernisierung seines Atomwaffenarsenals bereits viel früher in Angriff genommen als die USA. Allerdings stehen entsprechende Vorhaben neuerdings unter dem Vorbehalt eingeschränkter finanzieller Möglichkeiten. Ungeachtet dessen ist Moskau weit vorangeschritten, alle Raketen, die noch aus der Sowjetära stammen, durch neue zu ersetzen. Landgestützte strategische Raketen werden silobasiert oder mobil für Straße oder Schiene stationiert oder geplant und tragen entweder nur einen oder mehrere nukleare Sprengköpfe. Die Anzahl dieser Trägersysteme dürfte nicht ansteigen, auch aus Kostengründen. Russland möchte dies aber kompensieren, indem es neue Raketentypen, wie die bereits getestete RS-24 „Sarmat“, mit womöglich bis zu 24 Sprengköpfen ausstattet. Ähnlich sieht die Situation bei Russlands seegestützten nuklearen Streitkräften aus. Hier werden U-Boote und auf ihnen stationierte ballistische Raketen erneuert und mit mehr nuklearen Sprengköpfen bestückt. Auch Russlands Fernbomberflotte einschließlich luftgestützter Marschflugkörper wird modernisiert. Besonders die vermehrte Ausstattung ballistischer Raketen mit Mehrfachsprengköpfen ist tendenziell destabilisierend. Denn dies bedeutet, dass eine hohe Anzahl von Kernwaffen auf eine kleine Anzahl von Zielen vereint ist. Um also in einer Krise nicht Gefahr zu laufen, viele eigene Atomwaffen infolge eines gegnerischen Überraschungsangriffs zu verlieren könnte Moskau versucht sein, sein Nukleararsenal relativ frühzeitig zum Einsatz zu bringen. ${ }^{9}$

Aus europäischer Sicht besonders besorgniserregend sind Russlands nukleare Rüstungen bei den nicht-strategischen Waffen. In diesem Bereich werden neue land-, seeund luftgestützte Systeme eingeführt. Nicht nur verstößt Moskau mit einem neuen landgestützten Marschflugkörper allem Anschein nach gegen den INF-Vertrag. ${ }^{10}$ Vielmehr besteht im Westen die Sorge, dass Moskau in seiner Militärstrategie den frühen, aber begrenzten Einsatz nichtstrategischer Kernwaffen vorsieht. Dies entweder im Sinne einer Absicherung eines fait-accompli nach einem eigenen

8 Vgl. US Department of Defense 2018.

9 Vgl. Kristensen/Norris 2017; Rudischhauser 2018.

10 Vgl. Gordon, Michael R.: Russia Deploys Missile, Violating Treaty and Challenging Trump, New York Times, 14.2.2017. 
konventionellen Angriff auf kleinere NATO-Staaten, oder, um einen Konflikt mit der NATO, aus welchen Gründen er auch entstanden sein mag, zu seinen Gunsten zu beenden. Das dahinterliegende Kalkül wäre in jedem Fall, dass die NATO nach einem begrenzten nuklearen Einsatz Russlands nicht selbst nuklear eskalierte, sondern kapitulierte. ${ }^{11}$

Atomare Aufrüstung ist hingegen schon lange keine hauptsächlich amerikanisch-russische Domäne mehr. Vielmehr entwickelt sich vor allem in Asien bereits seit langem eine nukleare Rüstungsdynamik, die eine Menge Potenzial für Instabilität mit sich bringt.

China hat sich über lange Zeit eine nukleare Zurückhaltung auferlegt. Seit einigen Jahren wird Chinas Nuklearpolitik jedoch dynamischer. Zwar bleibt Peking beim deklaratorischen Verzicht auf den nuklearen Ersteinsatz. Doch wird China sein Atomwaffenarsenal quantitativ und qualitativ weiterentwickeln. Ziel ist eine robustere nukleare Zweitschlagfähigkeit in einem sich wandelnden Umfeld. Momentan wird das Arsenal landgestützter Raketen erneuert. Ältere, meist silobasierte Raketen mit Flüssigtreibstoffantrieb werden durch neuere, straßenbewegliche Systeme mit Feststoffantrieb ersetzt. Diese sind überlebensfähiger, schneller einsatzbereit und zielgenauer. Ferner werden einige der chinesischen Langstreckenraketen mit Mehrfachsprengköpfen ausgestattet. Überdies modernisiert China seine seegestützte nukleare Abschreckungskomponente. Schließlich sollen bereits im Test befindliche Hyperschallgleiter China in die Lage versetzen, amerikanische Raketenabwehrsysteme überwinden zu können. ${ }^{12}$

Der Wechsel von silogestützten Flüssigtreibstoffraketen zu straßenbeweglichen Festtreibstoffraketen soll die Überlebensfähigkeit der chinesischen Nuklearstreitkräfte stärken. Zugleich werden diese Systeme ohne aufwändiges Auftanken jederzeit einsatzbereit sein. Im gleichen Masse bedeutet der Aufbau einer nuklearen Komponente auf U-Booten ebenfalls eine Verbesserung der Überlebensfähigkeit chinesischer Nuklearwaffen. Gleichzeitig könnte damit künftig eine Abkehr von der bisherigen Politik der getrennten Lagerung von Raketen und nicht zusammengesetzten Sprengköpfen verknüpft sein. Die vermehrte Konzentration auf die Mehrfachsprengkopftechnologie (MIRV) stärkt zwar einerseits die chinesische Zweitschlagfähigkeit, da auch dann noch ein hoher Schaden für den Angreifer angedroht werden kann, wenn nur wenige der eigenen Raketen einen gegnerischen Erstschlag überleb-

11 Vgl. Kroenig 2015 sowie der Aufsatz von Kroenig in diesem Heft. 12 Vgl. Xuanzun, Liu: China tests hypersonic aircraft that can 'break any missile defense system', Global Times, 5.8.2018. ten. Jedoch stellen Raketen mit mehreren Sprengköpfen für den Angreifer in einer Krise sehr lohnende Ziele dar, da mit einem eigenen Sprengkopf mehrere gegnerische zerstört werden können, was in Kriseninstabilität resultieren kann. ${ }^{13}$

Indien verfolgt eine Politik der nuklearen Minimalabschreckung und des Verzichts auf den Ersteinsatz von Atomwaffen. Die indische politische Elite hat sich aber verpflichtet, eine komplette Triade aus land-, see- und luftgestützten Kernwaffen aufzubauen. Indien arbeitet intensiv an der Entwicklung neuer landgestützter Raketen wie der Agni-5, die Ziele in ganz China treffen könnte. Überdies hat Indien inzwischen sein zweites U-Boot, das mit ballistischen Raketen ausgerüstet ist, in Dienst gestellt. Auch arbeitet Indien an der Mehrfachsprengkopftechnologie, was jedoch noch einige Zeit in Anspruch nehmen dürfte. Delhi entwickelt seine Nuklearwaffen weiter, um seine derzeit hauptsächlich auf nuklearfähigen Flugzeugen basierende Abschreckungsfähigkeit gegenüber dem zahlenmäßig und qualitativ nuklear überlegenen China zu verbessern. Damit manövriert sich Delhi jedoch zunehmend in Zielkonflikte: Was glaubwürdig ist gegenüber China, ist nicht minimal gegenüber Pakistan; und was minimal ist gegenüber Pakistan, ist nicht glaubwürdig gegenüber China.

Obgleich Pakistan sich zumindest rhetorisch an einer nuklearen Minimalabschreckung orientiert, wächst sein Atomwaffenarsenal derzeit am schnellsten von allen Kernwaffenbesitzern. Islamabad behält sich den Ersteinsatz von Kernwaffen beispielsweise im Falle eines indischen konventionellen Angriffs vor. Mit seinem Erzfeind Indien sieht sich Pakistan in einem Wettlauf um die Modernisierung nuklearer Trägersysteme. Dies könnte Instabilität zwischen beiden Ländern befördern. Unter dem Gesichtspunkt der Stabilität erscheint es besonders problematisch, dass Pakistan sich immer mehr mit Fragen der nuklearen Kriegführung befasst und taktischen Kernwaffen zum Ausgleich gegenüber einer wachsenden indischen Überlegenheit bei konventionellen Waffen eine immer größere Rolle zuweist. ${ }^{14}$

Nordkorea ist ein weiterer nuklearer Unsicherheitsfaktor. Das Land kann vermutlich einfache nukleare Sprengsätze bauen. Umstritten bleibt, ob Pjöngjang fähig ist, diese auf ballistischen Raketen zu platzieren. In jedem Fall fühlen sich Südkorea und Japan sowie weitere Staaten in der Nachbarschaft durch Nordkoreas Atom- und Raketenpotenzial massiv bedroht. Auch die USA könnten in Pjöngjangs Visier geraten, was wiederum amerikanische

13 Vgl. Kristensen/Norris 2018a.

14 Vgl. Hall 2014, 363f.; Khan Z./Khan A. 2016. 
Beistandszusagen für Verbündete in der Region tendenziell schwächt. Ob die Absichtserklärung zwischen US-Präsident Trump und dem nordkoreanischen Führer Kim Jong Un vom Juni 2018 in einer kompletten nuklearen Abrüstung münden wird, kann bezweifelt werden. ${ }^{15}$

Nicht nur zwischen den USA und der NATO einerseits und Russland andererseits findet also eine an Intensität zunehmende nukleare Rüstungsdynamik statt. Vielmehr ist Ähnliches auch in Asien zu beobachten, wobei die USA und Russland selbst auch Bestandteil der dortigen Prozesse sind. So erfolgen Chinas Bemühungen, seine nukleare Zweitschlagfähigkeit zu steigern, im Angesicht verbesserter amerikanischer Fähigkeiten bei der Raketenabwehr. Chinas Aufrüstung beunruhigt wiederum seinen Nachbarn Indien, der entsprechend nachzieht, was wiederum Indiens Erzfeind Pakistan als Vorlage für eine eigene nukleare Aufrüstung dient. Zusammen ergibt dies ein Bild einer mit einer wachsenden Komplexität behafteten nuklearen Dynamik, die ungesteuert in einer hohen Instabilität münden kann. In politischen Krisensituationen wächst damit die Gefahr, dass Kernwaffen zum Einsatz kommen. ${ }^{16}$

Dieser Befund wird noch durch die Beobachtung verstärkt, dass technologische Entwicklungen die Fundamente der nuklearen Abschreckung erodieren. Denn die Überlebensfähigkeit von Kernwaffen, zentrale Voraussetzung für die Aufrechterhaltung einer gesicherten nuklearen Zweitschlagfähigkeit, ist zunehmend in Gefahr. Ein Grund dafür sind massiv gesteigerte Zielgenauigkeiten von Raketen, auch solchen, die auf U-Booten stationiert sind, infolge besserer Datenverarbeitung. Nukleare Angriffe auf gegnerische Raketenbasen mit relativ geringen Opferzahlen könnten somit zu einer realistischen Option werden. Ferner können mobile Atomraketen heute sehr viel besser aufgespürt und verfolgt werden, als dies noch vor wenigen Jahren möglich war. Auch das Aufspüren von U-Booten wird radikal verbessert. Beides unterläuft zusammen mit der gesteigerten Zielgenauigkeit ballistischer Raketen die gesicherte nukleare Zweitschlagfähigkeit. Zu erwarten ist daher eine, auch von der Technologieentwicklung getriebene, sich intensivierende nukleare Rüstungsdynamik. ${ }^{17}$

15 Vgl. Kristensen/Norris 2018b; der Text der Vereinbarung von Singapur ist abgedruckt in: The Times, 12.06.2018.

16 Vgl. eine frühere Zusammenfassung der derzeitigen nuklearen Rüstungsdynamik: Thränert 2016.

17 Vgl. Lieber/Press 2017.

\section{Zentrale Unterschiede zwischen dem Kalten Krieg und heute}

Die derzeitigen nuklearen Entwicklungen, die viel Potenzial für Instabilitäten in sich bergen, verlangen also nach einer Rüstungskontrolle. Nötig ist eine Politik, die auf der Basis der Einsicht, dass die Interessen des Gegners im wohlverstandenen Eigeninteresse immer mitgedacht werden müssen, das gemeinsame Überleben im Nuklearzeitalter sichert. Warum gelingt dies nicht? Einige allgemeine Antworten bieten sich zunächst an. So verblasst die Wirkung der Atombombenabwürfe über Hiroshima und Nagasaki. Die aktuelle Politikergeneration und auch die Öffentlichkeit haben die entsprechenden Bilder offenbar wenig präsent. Anders als früheren Politikern sitzt heutigen Entscheidungsträgern auch nicht mehr der Schreck über die nur mit viel Glück überstandene KubaKrise im Nacken. In den Regierungsbürokratien ist das Thema der nuklearen Rüstungskontrolle seit Jahren verwaist, sodass von dort keinerlei Impulse kommen. In der NATO ist offenbar seit Ende des Kalten Krieges vergessen worden, wie Rüstungskontrollverhandlungen zu führen wären. Schließlich fehlt vielen nuklearen Akteuren, vor allem in Asien, jegliche Erfahrung mit der Erarbeitung und Umsetzung von Rüstungskontrollabkommen für Kernwaffen. Statt sich mit den schwierigen Problemen der notwendigen Stabilisierung nuklearer Abschreckung auseinanderzusetzen, überwiegt der national geprägte Stolz, über die stärkste aller Waffen zu verfügen. Atomwaffen werden als Instrument eigener Stärke begriffen, nicht als Waffen, die aufgrund ihrer schier unvorstellbaren Zerstörungswirkung einen Sinn für gemeinsame Verantwortung erforderlich machen. Dieser Mangel an gemeinsamer Verantwortung hat womöglich sogar damit zu tun, dass in den Köpfen einiger Strategen neue technische Entwicklungen den selektiven Einsatz von Kernwaffen mit relativ geringen Opferzahlen künftig zulassen könnten.

Drei weitere wichtige Gründe, warum nukleare Rüstungskontrolle nahezu zum Erliegen gekommen ist, sollen im Folgenden einer näheren Betrachtung unterzogen werden: Der Mangel an Führung im derzeitigen internationalen System; die Ablösung der einstigen nuklearen Bipolarität durch eine nukleare Multipolarität; und die wachsende Verknüpfung der Problematik der Kernwaffen mit nichtnuklearen militärischen Entwicklungen. 


\subsection{Mangel an Führung}

Ein komplexes Politikkonzept wie das der nuklearen Rüstungskontrolle verlangt nach politischer Führung. Sie wurde in der liberalen Weltordnung von den USA bereitgestellt. Diese Ordnung entsprach den nationalen amerikanischen Interessen, gab aber genügend Raum für die Berücksichtigung der Interessen der anderen Akteure. Nukleare Rüstungskontrolle mit dem Ziel, zu regelbasierten Lösungen zu kommen, die umfassende, vernichtende Atomkriege möglichst ausschlossen, war Teil der liberalen Weltordnung.

Derzeit beobachten wir den relativen Niedergang Amerikas, also derjenigen Macht, die allein dazu in der Lage wäre, einen globalen Ordnungsanspruch durchzusetzen. ${ }^{18}$ Diese Entwicklung zeichnete sich bereits spätestens mit der Präsidentschaft Barack Obamas ab. Im nuklearen Bereich war dessen Vision eines „Global Zero“ für alle Kernwaffen vielleicht der vorerst letzte Versuch Amerikas, eine auf Kooperation angelegte nukleare Ordnung durchzusetzen. Auch wenn sich die Abschaffung aller Atomwaffen nicht als konkretes politisches Ziel eignete, setzte Washington damit doch ein wichtiges Signal: Es war bereit, die Probleme des nuklearen Zeitalters gemeinsam mit anderen so anzugehen, dass befriedigende Lösungen für alle möglich würden.

Davon kann heute kaum noch die Rede sein. Auch wenn die USA unter Donald Trump die Rüstungskontrolle nicht komplett über Bord geworfen haben, so spielt sie doch kaum noch eine Rolle. Im Vordergrund stehen vielmehr die Bemühungen um die Modernisierung des eigenen Kernwaffenpotenzials. ${ }^{19}$ Dies ist sicher zum Teil der Tatsache geschuldet, dass Russland seinerseits Rüstungskontrolle gering schätzt und Verträge nicht einhält. Die amerikanische Haltung entspricht aber auch dem Verlangen nach Aufrechterhaltung von Handlungsfähigkeit in einem von zunehmender Unsicherheit geprägten internationalen System. Die Aushandlung komplizierter, langwieriger Rüstungskontrollverträge ist nicht im Sinne Trumps. Vielmehr geht es ihm vorwiegend um die Durchsetzung dessen, was er unter „America First“ versteht. Dieses Ansinnen ist hauptsächlich innenpolitisch motiviert. Konkrete Problemlösungen sind nicht das Ziel, sondern die permanente Mobilisierung der eigenen Anhängerschaft. ${ }^{20}$ Diese

18 Vgl. Münkler 2018.

19 Die Nuclear Posture Review (NPR) der Trump-Administration spricht sich zwar für die Fortsetzung gewisser Rüstungskontrollelemente aus, doch spielt die Rüstungskontrolle in der NPR eine nur untergeordnete Rolle, vgl. Thränert 2018.

20 Vgl. Maull, Hanns W.: Bilder verdrängen Fakten, Frankfurter Allgemeine Zeitung, 26.6.2018. wiederum ist nur gegeben, wenn amerikanische Vorteile durchgesetzt werden. Der Trump-Administration geht es weniger darum, in einer internationalen Gemeinschaft für alle vorteilhafte Kompromisse zu erzielen. Ziel ist es vielmehr, in einer auf Konkurrenz angelegten internationalen Arena auf der Siegerseite zu sein. ${ }^{21}$ Eine auf Ausgleich angelegte Rüstungskontrollpolitik ist damit nicht vereinbar. Zumal die USA bei bestimmten technischen Entwicklungen, die wichtige Vorteile in der nuklearen Konkurrenz mit anderen Kernwaffenmächten versprechen, wie verbesserte Zielgenauigkeiten von Raketen oder effektivere Mittel zur Aufklärung und Verfolgung mobiler landgestützter Raketen oder U-Booten, führend sind. Insofern liegt es jedenfalls aus einer Trump-Perspektive näher, die sich bietenden Vorteile zum eigenen Nutzen auszuspielen, anstatt sie womöglich am Verhandlungstisch aufzugeben.

Auch von Russland sind keine Führungsleistungen im Sinne der nuklearen Rüstungskontrolle zu erwarten. Wie Herfried Münkler treffend feststellte, beruht Russlands Macht nicht darauf, zu politischer Stabilität beizutragen, sondern auf seiner Fähigkeit, die Projekte anderer zunichte zu machen. ${ }^{22}$ Auch bei der Rüstungskontrolle verhält sich Moskau destruktiv, wie seine offensichtlichen Verstöße gegen das INF-Abkommen zeigen. Überdies ist Russlands internationale Politik ebenso wie diejenige der USA vorwiegend innenpolitisch getrieben. Die Konstruktion und Pflege eines Bilds vom Westen, der Russland permanent alles Bösen in der Welt beschuldigt, dient der Mobilisierung patriotischer Gefühle, was wiederum der Stabilisierung des Systems Putins entgegenkommt. ${ }^{23}$ Insofern nutzt Putin eine Drohpolitik gegenüber dem Westen, nicht aber eine auf Kooperation angelegte nukleare Rüstungskontrolle mit den USA und womöglich weiteren Kernwaffenstaaten. Ferner ist das Atomwaffenarsenal, das sich wie geschildert in einem umfassenden Erneuerungsprozess befindet, eines der Kronjuwelen russischen Großmachtanspruchs, die man sich nicht wegverhandeln lassen will, insbesondere dann, wenn die wirtschaftliche Stärke Russlands abnimmt.

Allen anderen Kernwaffenstaaten fehlt es an jeglicher Erfahrung in Sachen nuklearer Rüstungskontrolle. Das aufstrebende China konzentriert sich ebenso wie die USA und Russland auf die Durchsetzung eigener Interessen. Eine auf Ausgleich angelegte nukleare Rüstungskontrollpolitik bleibt ihm weitgehend fremd. Zudem befürchtet China, dass die mit nuklearer Rüstungskontrolle einher-

21 Vgl. Brands 2017/2018, 15.

22 Vgl. Münkler 2018.

23 Vgl. Adomeit 2017. 
gehende verbesserte Transparenz die Überlebensfähigkeit seiner relativ kleinen nuklearen Zweitschlagkomponente gefährden könnte. ${ }^{24}$ Indien möchte zwar als verantwortlicher nuklearer Akteur anerkannt werden, sich aber im Angesicht seines großen Nachbarn China keine nuklearen Beschränkungen auferlegen. Schließlich ist Pakistan nicht an Rüstungskontrolle interessiert, solange Neu-Delhi in dieser Hinsicht keine Anstalten macht. ${ }^{25}$

Derzeit spricht wenig dafür, dass sich an dieser ernüchternden Konstellation Wesentliches ändert. Selbst eine Abwahl Donald Trumps wird die USA nicht wieder gleichsam automatisch auf den Tugendpfad der nuklearen Rüstungskontrolle zurückführen. Die Konzentration auf eng definierte nationale Interessen entspricht einem weit verbreiteten Grundgefühl eines Amerikas, das dabei ist, seiner Führungsrolle in der Welt verlustig zu gehen. Abgesehen davon ist das politische System derartig polarisiert und blockiert, dass man sich die langjährige Aushandlung nuklearer Rüstungskontrollabkommen nebst Ratifikation mit der erforderlichen 2/3 Mehrheit im Kongress schon gar nicht mehr vorzustellen vermag. ${ }^{26}$ Die Tatsache, dass Russland den INF-Vertrag offenbar verletzt, ist zudem Wasser auf die Mühlen derjenigen in Washington, die jegliche Rüstungskontrolle grundsätzlich ablehnen. Gleichzeitig sind die objektiven Problemlagen hinsichtlich der nuklearen Rüstungskontrolle noch viel komplexer geworden, als zu Zeiten des Kalten Krieges.

\subsection{Die nukleare Bipolarität ist einer nuklearen Multipolarität gewichen}

Nukleare Rüstungskontrollabkommen zwischen den USA und der Sowjetunion und später Russland orientierten sich aus Gründen der Demonstration politischer Gleichrangigkeit am Prinzip der Parität. Die entsprechenden Verträge basierten auf gemeinsamen Obergrenzen für bestimmte nukleare Waffensysteme. Aufgrund der unterschiedlichen Ausprägung der Atomwaffenarsenale - die USA sind eine maritime Macht, die Sowjetunion und heute Russland ist eine Landmacht - wurden im Rahmen der Obergrenzen bestimmte Spielräume festgelegt und auch die vereinbarten Zählregeln erlaubten eine gewisse Flexibilität. Am Prinzip der strategischen Parität wurde jedoch nicht gerüttelt. Dies war insofern möglich, weil Washington wie auch Moskau ihre Sicherheit hauptsächlich als

24 Vgl. Cunningham/Fravel 2015.

25 Vgl. Hall 2014.

26 Vgl. Thompson 2018. aufeinander bezogen betrachteten und weitere Nuklearmächte nur eine untergeordnete Rolle spielten. ${ }^{27}$

Auch heute besitzen die USA und Russland nach wie vor etwa $90 \%$ aller Kernwaffen weltweit. Doch hat sich die strategische Lage für beide insofern grundlegend geändert, als weitere Staaten Atomwaffen- und Raketenarsenale aufbauen, die aus amerikanischer wie auch russischer Perspektive für die jeweilige nationale Sicherheit von strategischer Bedeutung sind. Daher sind bilaterale Übereinkünfte über die Festlegung gemeinsamer Obergrenzen für Atomwaffen oder anderer Systeme, die für die nukleare Abschreckung relevant sind, wie Raketenabwehr, heute weder aus amerikanischer noch aus russischer Sicht identisch mit der Herstellung nationaler Sicherheit. Zwei Beispiele mögen dies verdeutlichen: Die amerikanische Kündigung des ABM-Vertrages und die vermutlichen russischen Verstöße gegen den INF-Vertrag.

$\mathrm{Zu}$ Beginn der siebziger Jahre hatten sich die USA und die Sowjetunion auf den ABM-Vertrag geeinigt, der die strategischen Raketenabwehrsysteme auf je zwei, später aufgrund eines weiteren Vertragsprotokolls nur noch auf je eine Verteidigungsstellung begrenzte. Sinn und Zweck dieses Abkommens war die gegenseitige Akzeptanz von Verwundbarkeit. Da beide Seiten weitgehend auf Schadensbegrenzungsoptionen verzichteten, galt, dass keine Seite hoffen konnte, einen Atomkrieg mit akzeptablen Schäden zu überstehen. Dies sollte die nukleare Abschreckung stärken und einen umfassenden Atomkrieg möglichst unwahrscheinlich machen. ${ }^{28}$

Unter dem Eindruck der Terroranschläge vom 11. September 2001 und der Erkenntnis, dass eine Reihe von Staaten dabei war, sich weitreichende Raketen und womöglich auch ABC-Waffen zu verschaffen, war das Prinzip der Akzeptanz von Verwundbarkeit für die USA nicht mehr tragbar. Daher kündigte der damalige USPräsident George W. Bush im Dezember 2001 mit Wirkung vom 30. Juni 2002 den ABM-Vertrag mit Verweis auf die Austrittsklausel und die dort verankerte Möglichkeit, aufgrund veränderter nationaler Sicherheitslagen das Abkommen $\mathrm{zu}$ verlassen. Er wies dabei ausdrücklich darauf hin, dass sich dieser Schritt nicht gegen Russland richtete. Aber in Zeiten, in denen die größten Bedrohungen von Terroristen und so genannten „Schurkenstaaten“ wie Nordkorea oder Iran ausgingen, die mit Raketen Amerika angreifen könnten, sei es seine Aufgabe als

27 Die Sowjetunion strebte zwar schon während der achtziger Jahre die Berücksichtigung der britischen und französischen Nukleararsenale bei den INF-Verhandlungen mit den USA an, scheiterte damit jedoch. Offenbar war dies aus Moskauer Sicht damals akzeptabel. 28 Vgl. Garthoff 1978. 
Oberkommandierender, Maßnahmen zu ergreifen, um die amerikanische Bevölkerung zu schützen. ${ }^{29}$ Die NATO folgte diesem amerikanischen Schritt, indem sie auf ihrem Lissabonner Gipfel 2010 beschloss, eine Raketenabwehr zum Schutz von NATO-Territorien und Bevölkerungen zu errichten. Diese richte sich jedoch gegen die Verbreitung von Raketen, nicht gegen Russland. ${ }^{30}$

Die USA weisen eine Beziehung der gegenseitigen nuklearen Verwundbarkeit, wie seinerzeit mit Blick auf die Sowjetunion, gegenüber heutigen regionalen nuklearen Herausforderern grundsätzlich ab. Dies betrifft beispielsweise Nordkorea, aber auch China. Schadensbegrenzungsmöglichkeiten gegenüber solchen nuklearen Mächten werden als notwendig erachtet, um die Glaubwürdigkeit der Beistandszusagen gegenüber Allliierten nicht zu unterlaufen. Daher ist Raketenabwehr für die USA zu einem tragenden Element regionaler Abschreckungsarchitekturen geworden. ${ }^{31}$

Russland hingegen sieht amerikanische und NATORaketenabwehrprojekte als bedrohlich für die eigene nationale Sicherheit an. Moskau fürchtet die sich allmählich entwickelnde, grundsätzlich unbegrenzte amerikanische Raketenabwehrarchitektur, die die russische Fähigkeit zur Führung eines nuklearen Zweitschlages und damit die russische Abschreckungskapazität unterlaufen könnte. Versuche, zwischen den USA und NATO einerseits und Russland andererseits zu einem Ausgleich oder sogar zu einer Zusammenarbeit bei der Raketenabwehr zu kommen, verliefen im Sande. ${ }^{32}$

Dieses Beispiel zeigt, dass Einflüsse dritter staatlicher (und nichtstaatlicher) Akteure heute zentral auf die Problematik der Stabilität zwischen den USA und Russland einwirken. Während es für Washington wegen der weltweiten Verbreitung von Raketentechnologie nicht möglich ist, Beschränkungen bei der Raketenabwehr zu akzeptieren, wird Moskau nicht bereit sein, mit den USA Vereinbarungen über die nukleare Rüstungskontrolle zu treffen, die die Raketenabwehr ausschließen.

29 Siehe U.S. Withdrawal From ABM Treaty: President Bush's remarks and U.S. Diplomatic Notes, Arms Control Today, February 2002; www.armscontrol.org/act/2002_01_02/docjanfeb02.

30 Vgl. NATO: Brussels Summit Declaration Issued by the Heads of State and Government participating in the meeting of the North Atlantic Council in Brussels 11-12 July 2018, https://www.nato.int/cps/ en/natohq/official_texts_156624.htm; sowie NATO: Lisbon Summit Declaration Issued by the Heads of State and Government participating in the meeting of the North Atlantic Council in Lisbon 19 20 November 2010, https://www.nato.int/cps/en/natohq/official_ texts_68828.htm.

31 Vgl. Roberts 2016, $82 \mathrm{ff}$.

32 Vgl. Zadra 2014.
Ein zweites Beispiel betrifft den INF-Vertrag. Der damalige sowjetische Generalsekretär Michail Gorbačev hatte im Rahmen der entsprechenden Verhandlungen zunächst angeboten, alle bodengestützten nuklearen Mittelstreckenwaffen in der europäischen Zone abzurüsten. Die Sowjetunion hatte solche Systeme, vorwiegend SS-20, jedoch auch in ihrem asiatischen Teil stationiert. Diese waren zur Abschreckung Chinas vorgesehen. Da Gorbačev unbedingt eine Vereinbarung mit den USA erzielen wollte, war er später bereit, eine globale Eliminierung aller landgestützten Mittelstreckenflugkörper zu akzeptieren. ${ }^{33}$

Heute könnte Russland ähnliche Zugeständnisse nicht mehr machen. Bereits während seiner Rede vor der Münchner Sicherheitskonferenz im Februar 2007 wies der russische Präsident Vladimir Putin darauf hin, dass inzwischen eine ganze Reihe von Staaten wie Nordkorea, Indien, Iran, Pakistan und Israel Raketen in genau jenem Reichweitenspektrum entwickelten, in dem die USA und Russland im Rahmen des INF-Vertrages gänzlich auf landgestützte Flugkörper verzichtet hatten. ${ }^{34}$

Das russische Unbehagen mit dem INF-Abkommen hat sich inzwischen massiv gesteigert. Die Aufrüstungsprozesse an Russlands Südflanke spielen dabei eine wichtige Rolle. Moskau ist ebenso besorgt über die chinesische nukleare Aufrüstung, wie es die gesamte nukleare Rüstungsdynamik in Asien als Bedrohung ansieht. ${ }^{35}$ Dies mag ein Grund sein, warum - laut amerikanischen Quellen Russland den INF-Vertrag im Zuge der Stationierung eines neuen landgestützten Marschflugkörpers verletzt. ${ }^{36}$ Ziel wäre es, mittels nicht-strategischer Kernwaffen für den asiatischen Schauplatz eine glaubwürdige Abschreckung zu errichten.

Beide Beispiele zeigen: In einer multipolar geprägten nuklearen Welt wirken Bedrohungen auf die USA und Russland ein, die nicht in direktem Zusammenhang mit dem jeweiligen Gegenüber stehen, sondern von Dritten ausgehen. Dies hat zur Folge, dass bilaterale Abkommen entweder gekündigt, oder verletzt werden. Neue bilaterale Vereinbarungen zu treffen ist dadurch doppelt schwierig: Zum einen, weil die Kündigung von Abkommen und mehr noch deren Verletzung Vertrauen zerstört; und zweitens, weil es nahezu unmöglich geworden ist, die in einem multipolaren nuklearen Umfeld sich sehr unterschiedlich entwickelnden Sicherheitsbedürfnisse in

33 Vgl. Risse-Kappen 1988, 133 und 173.

34 Vgl. Putin's Prepared Remarks at 43rd Munich Conference on Security policy, Washington Post, 10.2.2007.

35 Vgl. Kühn/Péczeli 2017, 73 f.

36 Vgl. Gordon, Michael R.: Russia Deploys Missile, Violating Treaty and Challenging Trump, New York Times, 14.2.2017. 
einem bilateralen Abkommen unter Berücksichtigung des Prinzips der Parität für beide Seiten befriedigend zu erfassen.

\subsection{Es geht nicht mehr nur ums Nukleare}

Während des kalten Krieges konnten Kernwaffen im Rahmen von Rüstungskontrollvereinbarungen relativ isoliert betrachtet werden. Dies galt zumindest, nachdem sich die USA und die Sowjetunion auf den ABM-Vertrag und damit auf den Verzicht der umfassenden Weiterentwicklung von Raketenabwehrsystemen geeinigt hatten. Diese Vereinbarung ist hingegen heute hinfällig. Damals existierten bereits Trägersysteme, die für nukleare wie konventionelle Bewaffnung vorgesehen waren. Heute ist dieses Problem im Zuge neuer technologischer wie strategischer und doktrinärer Entwicklungen jedoch sehr viel virulenter. Die Grenzen nuklearer und nichtnuklearer Domänen verschwimmen zunehmend. Schließlich treten ganz neue Herausforderungen etwa im Cyberbereich hinzu, die während des Kalten Krieges noch kaum eine Rolle spielten, heute jedoch massiv auf die strategische Situation einwirken.

Anlässlich seiner Rede zur Lage der Nation 2018 stellte der russische Präsident Vladimir Putin neue, in der Entwicklung befindliche Waffensysteme vor, deren Zweck es vor allem sei, die amerikanische Raketenabwehr überwinden zu können. ${ }^{37}$ Dies zeigte einmal mehr, dass Russland die amerikanischen Raketenwehrprojekte als Gefährdung seiner Sicherheitsinteressen ansieht. Daher hat Russland - oft unter Hinweis auf die Präambel des Neu-Start Vertrages, in der die Bedeutung der Raketenabwehr für die strategische Stabilität hervorgehoben wird - immer wieder deutlich gemacht, dass es nicht zu weiteren nuklearen Rüstungskontrollverträgen bereit sei, die nicht auch die Raketenabwehr begrenzten. ${ }^{38}$ In den USA hingegen gehört die Raketenabwehr zu den wenigen Themen, bei denen sich Republikaner und Demokraten insofern grundsätzlich einig sind, als sie Schadensbegrenzungsoptionen im

37 Katzenberger, Paul: Rede zur Lage der Nation: Putin markiert den starken Mann - und protzt mit Superwaffen, Süddeutsche Zeitung, 1.3.2018; s. a. Cordesman 2018.

38 US Department of State: New START Treaty Text, zitiert aus der Präambel: "Recognizing the existence of the interrelationship between strategic offensive arms and strategic defensive arms, that this interrelationship will become more important as strategic nuclear arms are reduced, and that current strategic defensive arms do not undermine the viability and effectiveness of the strategic offensive arms of the Parties," https://www.state.gov/t/avc/newstart/c44126. htm.
Angesicht neuer nuklearer Bedrohungen, darunter etwa aus Nordkorea, für unbedingt notwendig erachten. ${ }^{39}$ Insofern ist es unwahrscheinlich, dass Washington in nächster Zeit zu Begrenzungen bei der Raketenabwehr bereit wäre.

Doch nicht nur Russland, auch China sieht die USRaketenabwehr als schädlich für die strategische Stabilität an. Dadurch könne die chinesische nukleare Abschreckung neutralisiert werden. Schon ein dünnes Abwehrschild mit wenigen Flugkörpern (Interzeptoren) könnte ausreichen, um Chinas relativ wenige nukleare Raketen abzufangen. Darüber hinaus fürchtet Peking ganz grundsätzlich einen Rüstungswettlauf im Weltraum, der zu gefährlichen Konsequenzen führen könne. ${ }^{40}$ Auch aus Sicht Chinas machte daher jegliche Rüstungskontrolle, die Kernwaffen begrenzte, nicht aber Raketenabwehr, keinen Sinn.

Neben der US-Raketenabwehr sorgen sich Moskau wie Peking gleichermaßen über amerikanische Programme zum Aufbau einer Fähigkeit für konventionelle weitreichende Schläge (Conventional Prompt Global Strike). Hierzu werden in den USA unter anderem moderne Hyperschallgleiter entwickelt, die jeden Punkt der Erde binnen einer Stunde erreichen können sollen. Ziel ist etwa, bevorstehende Terroranschläge oder einen nordkoreanischen Raketenangriff zu vereiteln. Zwar arbeiten sowohl Russland als auch China selbst an entsprechenden Waffensystemen, doch fürchten beide die amerikanische technische Überlegenheit auf diesem Gebiet. Aus russischer wie auch aus chinesischer Perspektive könnten die USA mit solchen Waffen Instrumente an die Hand bekommen, die es Washington - besonders, wenn sie im Zusammenhang mit der US-Raketenabwehr gesehen werden - gestatteten, die nukleare Zweitschlagfähigkeit Russlands und Chinas durch frühe konventionelle, strategische Präzisionsschläge zu unterlaufen. ${ }^{41}$ Wiederum handelt es sich bei den weitreichenden konventionellen Präzisionswaffen um einen Bereich, den Moskau wie auch Peking nicht aus der Rüstungskontrolle ausklammern wollten, sollte es denn je zu Verhandlungen kommen. Zugleich sieht Washington jedoch seine Projekte zum Aufbau von Conventional Prompt Global Strike hauptsächlich im Zusammenhang mit Bedrohungen, die nicht in erster Linie mit Russland oder China verknüpft sind.

Ferner wandelt sich das Verhältnis zwischen nuklearen und konventionellen Waffen. Während des kalten

39 Vgl. Karako 2017.

40 Vgl. Bin 2018.

41 Vgl. Acton, James M.: Conventional Prompt Global Strike and Russia's Nuclear Forces, Webseite der Carnegie Endowment, 4.10.2013; https://carnegieendowment.org/2013/10/04/conventional-promptglobal-strike-and-russia-s-nuclear-forces-pub-53213. 
Krieges herrschte auf beiden Seiten des Eisernen Vorhangs die Vorstellung einer Eskalationsleiter vor. Vor allem im russischen strategischen Denken ist dieses Konstrukt durch einen integrativen Ansatz des simultanen Vorgehens in verschiedenen Domänen ersetzt worden. Das russische Abschreckungsdenken basiert heute auf einem Mix von konventionellen Präzisionswaffen und Kernwaffen, wobei beide als strategische Waffen angesehen werden. Moskau kombiniert also den strategischen Effekt konventioneller Präzisionswaffen mit dem Abschreckungseffekt von Kernwaffen. Somit kann Russland bei einem Gegner vom ersten Augenblick eines Konflikts an nukleare Unsicherheit auslösen. Durch die beim Gegner verursachte Verunsicherung will Russland die Abschreckung stärken und mehr Optionen bei der Eskalationskontrolle erlangen. ${ }^{42}$

In der Tat konzentriert sich Russland auf die Entwicklung von konventionell wie nuklear nutzbaren Systemen wie Marschflugkörpern und ballistischen Raketen. Überdies sind Hyperschall-Marschflugkörper und boost-glide Systeme mit nuklearer wie konventioneller Bewaffnung gleichermassen künftig für die Bekämpfung besonders wertvoller Ziele auserkoren. Auch wenn diese Waffenarten in Russland, in den USA und weiteren Staaten noch einige Entwicklungszeiten benötigen werden, stellt sich vor diesem Hintergrund zunehmend die Frage, inwiefern eine auf strategische Kernwaffen konzentrierte Rüstungskontrolle noch Sinn machte. Zumal auch China eine wachsende Anzahl von Raketen mit kürzeren Reichweiten besitzt, die zum großen Teil mit konventionellen Sprengköpfen bestückt sind, aber auch nuklear bewaffnet werden können.

Schließlich können Cyberattacken künftig strategische Qualität erreichen. Der russische Verteidigungsminister Šoigu verglich solche Operationen von den zu erzielenden Effekten her schon mit dem Einsatz von Massenvernichtungswaffen.$^{43}$ Auch auf amerikanischer Seite ist man sich der Bedeutung nicht-nuklearer strategischer Angriffe bewusst. Daher halten sich die USA gemäß der von der Trump-Administration veröffentlichten Nuclear Posture Review nukleare Ersteinsätze im Angesicht solcher Angriffe vor. Auch wenn dies im Text nicht explizit so erwähnt wurde, ist dabei auch an Cyberattacken mit strategischer Wirkung gedacht worden. ${ }^{44}$ Solche Attacken könnten sich beispielsweise gegen Frühwarnsysteme richten. Dies könnte die nukleare Zweitschlagfähigkeit gefährden. Oder es könnte die Stromversorgung eines oder mehrerer Länder unterbrochen und damit nachhaltige Schäden verursacht

42 Vgl. Johnson 2018; Zysk 2018.

43 Vgl. Johnson 2018.

44 US Department of Defense 2018. werden, insbesondere dann, wenn die Kühlversorgung von Atomkraftwerken oder anderen kritischen Infrastrukturen nicht mehr sichergestellt werden könnte.

Die wachsende Verwundbarkeit von Satelliten, bodengestützten Radaren oder Flugzeugen, die für die Frühwarnung, Kommunikation und Überwachung benutzt werden, bereitet sowohl in den USA als auch in Russland und China Kopfzerbrechen. Insbesondere auch deswegen, weil diese Systeme sowohl für konventionelle wie auch nukleare Auseinandersetzungen vorgesehen sind. Insofern besteht die Gefahr einer ungewollten nuklearen Eskalation, sollten etwa Kommunikationssysteme in einem begrenzten Konflikt zur Unterdrückung gegnerischer konventioneller Operationen angegriffen werden, da dadurch zugleich die Fähigkeit zur Aufrechterhaltung einer nuklearen Zweitschlagfähigkeit zur Disposition stünde. ${ }^{45}$ Die weiter wachsende Bedeutung des Weltraums wird durch die Absicht der USA unterstrichen, eine neue Teilstreitkraft speziell für diesen Bereich einzurichten. Ziel ist, die amerikanische militärische Dominanz im Weltraum aufrechtzuerhalten. ${ }^{46}$ Der Schutz von Frühwarnund Kommunikationsfähigkeiten zur Führung konventioneller wie möglicherweise nuklearer Konflikte spielt dabei eine zentrale Rolle. Auch dies unterstreicht, wie sehr die Domänen konventionell, nuklear, und Weltraum bereits heute miteinander verwoben sind.

Kernwaffen galten lange als Waffen, die von ihrer unvergleichlichen Zerstörungswirkung her als kriegsentscheidend eingeschätzt wurden. Es gab eine klare Trennlinie $\mathrm{zu}$ allen anderen Waffenarten. Diese Trennlinie verschwimmt zunehmend in Zuge der Entwicklung moderner konventioneller Präzisionswaffen ebenso wie im Kontext der Entwicklung neuer Möglichkeiten im Cyberbereich. Auch die Raketenabwehr und neue militärische Fähigkeiten im Weltraum sind von wachsender strategischer Bedeutung. Insofern wird es mehr und mehr fraglich, inwiefern eine einzig auf Kernwaffen konzentrierte Rüstungskontrolle zu strategischer Stabilität beitragen könnte.

\section{Wie geht es weiter?}

Nukleare Rüstungskontrolle steht also vor einer Vielzahl sehr schwieriger, teilweise gänzlich neuer Herausforderungen. Zugleich ist auf diesem Politikfeld bis auf weiteres keine politische Führungsleistung der USA zu erwar-

45 Acton 2018.

46 Davenport, Christian/Lamothe, Dan: Pence details plan for creation of Space Force in what would be the sixth branch of the military, Washington Post, 9.8.2018. 
ten. Die Autokratien dieser Welt wie Russland und China dürften diese Lücke kaum füllen. Zugleich können wir es uns nicht leisten, die gegenwärtige Situation tendenziell destabilisierender Entwicklungen einfach fortzuschreiben. Atomkriege könnten sonst wahrscheinlicher werden.

Was benötigt wird, um diese bedrohliche Entwicklung zu stoppen, ist ein Diskurs über die heutigen Herausforderungen für die strategische Stabilität. Nukleare Zweitschlagfähigkeit spielt dabei zwar nach wie vor eine Rolle, doch sind die Kontexte heute sehr viel komplexer als noch während des Kalten Krieges. Es geht nicht mehr nur um zwei Akteure, sondern eine größere Anzahl; und nicht-nukleare Technologien wirken heute in vielen größerem Umfang auf strategische Stabilität ein als während der klassischen Ost-West Konfrontation.

Deutschland könnte zu einer Debatte über strategische Stabilität wichtige Beiträge leisten. Zunächst müsste es darum gehen, den eigenen Diskurs über nukleare Rüstungskontrolle neu zu organisieren. Es ginge dann nicht mehr um die Verwaltung bestehender Abkommen, sondern um Rüstungskontrolle als Teil eines strategischen Diskurses. Es müsste also gefragt werden, wie nukleare Abschreckung künftig so organsiert wird, dass durch Rüstungskontrolle Stabilität erreicht wird und dass das Scheitern der Abschreckung möglichst ausgeschlossen bleibt. Im Vordergrund sollte daher Stabilität in einer weiterhin nuklearen Welt und nicht die nukleare Abrüstung als Ziel per se stehen. Insofern müsste auch die bislang gängige Verknüpfung von Rüstungskontrolle und Abrüstung mit der Erfüllung der Pflichten gemäß Artikel VI des Nuklearen Nichtverbreitungsvertrages gelöst werden. Vielmehr geht es darum, den Kern der nuklearen Rüstungskontrolle wieder frei zulegen, wonach die Sicherheitsinteressen des Gegenübers im wohlverstandenen Eigeninteresse immer mitgedacht werden müssen.

In einem zweiten Schritt sollte der Diskurs mit europäischen und anderen gleichgesinnten Partnern intensiviert werden. Frankreich, aber auch Großbritannien sind dabei als europäische Atommächte von herausragender Bedeutung. Die Diskussion mit diesen Partnern sollte nicht von dem Anliegen geprägt sein, dass das Ziel einer nuklearwaffenfreien Welt nicht völlig aus dem Blick gerät. ${ }^{47}$ Solche Äußerungen mögen vom eigenen breiten Publikum dankbar aufgenommen werden. In London und Paris schrecken sie hingegen eher ab. Für beide sind Kernwaffen nun einmal - man mag das mögen oder nicht - auch Sta-

47 Außenminister Heiko Maas: „Die Zukunft der nuklearen Ordnung - Herausforderungen für die Diplomatie“, Berlin, 27.6.2018, https://www.auswaertiges-amt.de/de/newsroom/maas-fes-tiergarten-konferenz/2112704. tussymbole. Anstatt diese Partner zu verprellen, sollten sie lieber in die Verantwortung genommen werden, um einen dringend notwendigen Rüstungskontrolldiskurs zu beginnen. Das funktioniert hingegen nur, wenn die Existenz von Atomwaffen nicht grundsätzlich in Frage gestellt wird.

Da es im Kreise der EU-Mitglieder mit Österreich und auch Irland Länder gibt, die sich der Kernwaffenverbotskonvention verschrieben haben, eignet sich die EU ganz grundsätzlich nicht mehr als Forum für eine Wiederbelebung des nuklearen Rüstungskontrolldiskurses. Daher bietet es sich an, neue Formate zu schaffen, die interessierte Staaten über Europa hinaus einbeziehen. Diese Formate können auf verschiedenen Ebenen ansetzen. Denkbar wären zunächst informelle Gespräche unter Einbezug von Experten aus dem Bereich von Think Tanks (Track 1.5 Format), die zu Regierungsgesprächen weiterentwickelt werden könnten.

Letztlich wird es auf die grossen nuklearen Akteure wie die USA und Russland, aber auch China ankommen. Ohne ihre Beiträge kann nukleare Rüstungskontrolle nicht wiederbelebt werden. Hilfestellung bei der dringend notwendigen Reanimation dieses so wertvollen und für das gemeinsame Überleben so notwendigen Politikkonzepts könnte Deutschland gemeinsam mit gleichgesinnten Partnern hingegen schon leisten.

\section{Literatur}

Acton, James (2018): Escalation through Entanglement, International Security, 43 (1), 56-99.

Adomeit, Hannes (2017): Innenpolitische Determinanten der Putinschen Außenpolitik, Sirius, 1 (1), 33-52.

Arbatov, Alexey (2017): Understanding the US-Russia Nuclear Schism, Survival, 59 (2) 33-66, 40.

Bin, Li (2018): China's attitudes toward missile defense and its limitation, Bulletin of the Atomic Scientists, 74 (4), 243-247.

Brands, Hal (2017/2018): The Unexceptional Superpower: American Grand Strategy in the Age of Trump, Survival, 59 (6) 7-40.

Brodie, Bernard (1978): The Development of Nuclear Strategy, International Security, 2 (4) 65-83.

Cordesman, Anthony H. (2018): Putin and Russia's New Nuclear Weapons: Whoever Dies with the Most Toys Wins? Washington, D.C.: Center for Strategic and International Studies.

Cunningham, Fiona S./Fravel, M. Taylor(2015): Assuring Assured Retaliation, China's Nuclear Posture and U.S.-China Strategic Stability, International Security, 40 (2), 7-50.

Forndran, Erhard (1970): Rüstungskontrolle: Friedenssicherung zwischen Abschreckung und Abrüstung. Düsseldorf: Bertelsmann-Universitätsverlag.

Garthoff, Raymond (1978): SALT I: An Evaluation, World Politics, 31 (1), 1-25. 
Gray, Colin S./Payne, Keith (1980): Victory is Possible, Foreign Policy, (39) 14-27.

Hall, Ian (2014): The Requirements of Nuclear Stability in South Asia, The Nonproliferation Review, 21 (3-4), 355-371.

Johnson, Dave (2018): Russia's Conventional Precision Strike Capabilities, Regional Crisis, and Nuclear Thresholds. Livermore: Livermore Papers on Global Security, (3).

Karako, Thomas (Hrsg.) (2017): Missile Defense and Defeat, Considerations for the new Policy Review. Washington, D.C.: Center for Strategic and International Studies.

Khan, Zulfgar/Khan, Ahmad (2016): The Strategic Impasse over India's Doctrinal Restructuring, The Washington Quarterly, 39 (1), 139-157.

Kissinger, Hery A. (1974): Kernwaffen und Auswärtige Politik. München: Oldenbourg.

Krause, Joachim (2011): Nach Hiroshima. Die Entwicklung der Theorie des Nuklearkrieges, in: Handbuch Kriegstheorien, hrsg. von Thomas Jäger und Rasmus Bergmann, Wiesbaden: VS Verlag, 413-426.

Kristensen, Hans M./Norris, Robert S. (2017): Russian nuclear forces, Bulletin of the Atomic Scientists, 73 (2), 115-126.

Kristensen, Hans M./Norris, Robert S. (2018a) Chinese nuclear forces 2018, Bulletin of the Atomic Scientists, 74 (4), 289-295.

Kristensen, Hans M./Norris, Robert S. (2018b): North Korean nuclear forces 2018, Bulletin of the Atomic Scientists, 74 (1), 41-51.

Kroenig, Matthew (2015): Facing reality: NATO ready for a new cold war, Survival, 57 (1), 49-70.

Kühn, Ulrich/Péczeli, Anna (2017): Russia, NATO, and the INF-Treaty, Strategic Studies Quarterly, 11 (1) 66-99.

Lieber, Keir A./Press, Daryl G. (2017): The New Era of Counterforce. Technological Change and the Future of Nuclear Deterrence, International Security, 41 (4), 9-49.

Münkler, Herfried (2018): Eine neue Sicherheitsarchitektur für Europa? Einige politikstrategische Überlegungen, Merkur, 72 (830), 5-17.
Risse-Kappen, Thomas (1988): Null-Lösung. Entscheidungsprozesse zu den Mittelstreckenwaffen 1970-1987, Frankfurt a.M./New York; Campus Verlag.

Roberts, Brad (2016): The Case for U.S. Nuclear Weapons in the 21th Century. Stanford: Stanford University Press.

Roberts, Brad (2018): Das internationale Abkommen zum Verbot von Kernwaffen - Was tun?, Sirius, 2 (2), 170-177.

Rudischhauser, Wolfgang (2018): Putins Waffenshow, Nur heiße Luft aus Moskau?: Berlin: Bundesakademie für Sicherheitspolitik, Arbeitspapier zur Sicherheitspolitik, Heft 7.

Schelling, Thomas (1966): Arms and Influence. New Haven, CT: Yale University Press.

Senghaas, Dieter (1972): Aufrüstung durch Rüstungskontrolle. Stuttgart: Kohlhammer.

Steiner, Barry H. (1991): Bernard Brodie and the Foundations of American Nuclear Strategy. Lawrence, KS: University of Kansas Press.

Thompson, Jack (2018): Superpower Constrained, in: Strategic Trends 2018, hrsg. Von Oliver Thränert/Martin Zapfe. Zürich: Center for Security Studies, ETH Zürich, 11-28.

Thränert, Oliver (2016): A Nuclear World Out of (Arms) Control, in: Strategic Trends 2016, hrsg. von Oliver Thränert/Martin Zapfe. Zürich: Center for Security Studies, ETH Zürich, 65-82.

Thränert, Oliver (2018): Präsident Trumps Nuclear Posture Review, Sirius, 2 (2), 158-161.

US Department of Defense (2018): Nuclear Posture Review. Washington D.C.: DoD, https://www.defense.gov/News/ SpecialReports/2018NuclearPostureReview.aspx/

Wohlstetter, Albert (1959): The Delicate Balance of Terror, Foreign Affairs, 37 (2), 212-234.

Woolf, Amy F./Kerr, Paul K./Nikitin, Mary Beth D. (2018): Arms Control and Nonproliferation: A Catalog of Treaties and Agreements. Washington, D.C.: Congressional Research Service.

Zadra, Roberto (2014): NATO, Russia and Missile Defense, Survival, $56(4), 51-61$. 